\title{
IMECE2008-66664
}

\section{A GAUSSIAN PROCESS-BASED APPROACH FOR HANDLING UNCERTAINTY IN VEHICLE DYNAMICS SIMULATION}

\author{
Kyle Schmitt* \\ Dept. Mech. Engineering \\ University of Wisconsin, Madison, WI \\ Email: kpschmitt@wisc.edu \\ Mihai Anitescu \\ Mathematics and Computer Science Div. \\ Argonne National Laboratory \\ Email: anitescu@mcs.anl.gov
}

\author{
Justin Madsen \\ Dept. Mech. Engineering \\ University of Wisconsin, Madison, WI \\ Email: jcmadsen@wisc.edu \\ Dan Negrut \\ Dept. Mech. Engineering \\ University of Wisconsin, Madison, WI \\ Email: negrut@wisc.edu
}

\section{ABSTRACT}

Advances in vehicle modeling and simulation in recent years have led to designs that are safer, easier to handle, and less sensitive to external factors. Yet, the potential of simulation is adversely impacted by its limited ability to predict vehicle $d y$ namics in the presence of uncertainty. A commonly occurring source of uncertainty in vehicle dynamics is the road-tire friction interaction, typically represented through a spatially distributed stochastic friction coefficient. The importance of its variation becomes apparent on roads with ice patches, where if the stochastic attributes of the friction coefficient are correctly factored into real time dynamics simulation, robust control strategies could be designed to improve transportation safety.

This work concentrates on correctly accounting in the nonlinear dynamics of a car model for the inherent uncertainty in friction coefficient distribution at the road/tire interface. The outcome of this effort is the ability to quantify the effect of input uncertainty on a vehicle's trajectory and the associated escalation of risk in driving. By using a space-dependent Gaussian process, the statistical representation of the friction coefficient allows for consistent space dependence of randomness. The approach proposed allows for the incorporation of noise in the observed data and a nonzero mean for inhomogeneous distribution

${ }^{*}$ Address all correspondence to this author. of the friction coefficient. Based on the statistical model considered, consistent friction coefficient sample distributions are generated over large spatial domains of interest. These samples are subsequently used to compute and characterize the statistics associated with the dynamics of a nonlinear vehicle model. The information concerning the state of the road and thus the friction coefficient is assumed available (measured) at a limited number of points by some sensing device that has a relatively homogeneous noise field (satellite picture or ground sensors, for instance). The methodology proposed can be modified to incorporate information that is sensed by each individual car as it advances along its trajectory.

\section{INTRODUCTION}

During the 1970s increasing awareness of the theory of stochastic process, together with the wider availability of digital computers, brought to automatic engineers a new and powerful technique for treating the response of vehicles to the irregular undulations of roads [1]. Over the last decade, stochastic techniques and computing power have been harnessed further, allowing for high-fidelity real-time simulations of vehicles on roads with uncertain conditions. The most common application of spatial uncertainty quantification has been in modeling vehicles under ran- 
dom road excitation. Random ground excitation has been modeled with spatial homogeneous random processes, the output of a linear shaping filter to white noise, as in $[2,3]$. A stochastic road excitation assumption is used in [4] to monitor tire conditions and reduce tire vibration. One prevailing method for addressing spatial randomness is the method of Gaussian processes, which has been employed to model road surfaces for stationary [5] or nonstationary processes, the latter represented as a series of stationary process [6].

An unexplored, commonly occurring, spatially stochastic parameter in vehicle dynamics is the road-tire friction interaction. The importance of this variation is exhibited on roads with ice patches. The physical challenges of low friction coefficients and control challenges of driver misperception are cited in [7] as key causes for the escalation of risk in winter weather conditions.

The primary goal of this work is to devise an efficient and flexible methodology focused on addressing uncertainty in vehicle dynamics simulation; we will use icy road conditions as our inherently stochastic enviroment for testing and evaluation of our methodology. To this end, Gaussian processes are proposed to produce continuous high-fidelity models of icy terrain from a discrete set of known friction values (attained from satellite imagery, ground sensors, or information estimated by other vehicles [8,9]). A vehicle model is simulated on the constructed terrain to quantify the effect of ice patches on a vehicle's trajectory (compared to the deterministic case) and to quantify the escalated risk of spinout and oversteer. The proposed methodology is demonstrated in conjunction with two simulation environments. The first one draws on the MATLAB package, which is used to implement a simplified bicycle model, and the second one is the MSC.ADAMS/Car commercial software package, widely used in industry for vehicle dynamics simulation [10].

\section{PROPOSED METHODOLOGY}

In this work, modeling the friction coefficient at the wheelground contact draws on a Gaussian process approach to provide a consistent space distribution based on information available at a limited number of locations.

Other approaches for modeling randomness in the road surface or road-tire interaction do exist. One class of past approaches is based on homogeneous random processes [2, 3]. While these approaches model a large class of problems and may be useful in design and simulation, they are nonetheless not appropriate for situations where the variation of the road surface has large areas of coherence that are inhomogeneous, even if they may be stationary in terms of the uncertainty given the measured surface data. Another class of past approaches is the one that we call "spectral" Gaussian processes [5]. In these approaches, the properties of the surface are represented by their spatial Fourier transform with independent, normally distributed, coefficients. As a result, the distribution of the respective property is also
Gaussian at every point in space, which is also the case for our approach. Nonetheless, our approach, which is based on an initial specification of the covariance function, has two key advantages. The first advantage originates in the fact that spectral Gaussian process approaches cannot easily accommodate rapid variations in the properties of the surface, which is a well-known side effect of the Gibbs phenomenon. In the proposed approach, since both the representation of the Gaussian process and the data fitting procedure occur in real space, there is far more flexibility in dealing with such situations (which appear, for example, when one considers the limits of the road). Such difficulties may conceivably be overcome by spectral methods by using a different orthogonal basis defined only in the region of interest. However, the complexity and computational effort to generate such a basis may be far from trivial; and in fact such an approach has not, to our knowledge, been demonstrated. The second advantage of the proposed approach has to do with the fact that covariance function-based Gaussian process modeling is one of the prevalent methods for representing spatial uncertainty $[11,12]$. Therefore, road surface data will eventually be provided in a format compatible with this representation.

An approach that has recently generated major interest in uncertainty quantification of engineering systems has been the one of polynomials chaos expansions. While that approach is extremely flexible, it also requires approximating the states of the system in a polynomial basis that grows roughly as $n^{d}$, where $n$ is the polynomial degree used and $d$ is the dimension of the uncertainty space. Such an approximation is intractable for problems that are obtained by spatial discretization with uncertainty at each node, of the type that is treated here.

A key modeling decision is the selection of a covariance function. Various studies in geostatistics suggest that the squared exponential is a representative correlation function [11], and a variation of that function is will be used herein. Because the friction coefficients are naturally bounded between two extreme values (that of dry land $\mu_{d}$ and ice $\mu_{s}$, where $\mu_{d}>\mu_{s}$ and are selected from pg. 27 of [13]), the quantity modeled will be the logarithm of a ratio involving the friction coefficient by the Gaussian process. A function $f$ is introduced to provide $\mu$ everywhere as

$$
\mu=\mu_{s}+\left(\mu_{d}-\mu_{s}\right) * \frac{1}{1+e^{-f}} .
$$

Therefore,

$$
f=-\ln \left(\frac{\mu_{d}-\mu}{\mu-\mu_{s}}\right) \in(-\infty, \infty) .
$$

Herein, $f$ is assumed to be a field providing values at a $n$ node grid through a Gaussian process that is identified based on 
a $m$-node grid of measurement points. Typically, $n \gg m$. Considering the road flat (two-dimensional), at locations on $\mathbf{x}=\left(x_{1}, x_{2}\right)$ it is assumed that $f(\mathbf{x}) \sim G P\left(m(\mathbf{x}), k\left(\mathbf{x}, \mathbf{x}^{\prime}\right)\right)$. That is, the field $f(\mathbf{x})$ is defined as a Gaussian process with mean function $m(\mathbf{x})$ and covariance function $k\left(\mathbf{x}, \mathbf{x}^{\prime}\right)$ [14]. A degree one polynomial mean function is used here to account for a nonstationary spatial distribution in the $x_{1}$ and $x_{2}$ directions, while the covariance function is assumed a squared exponential. It should be noted here that Gaussian processes can be used to consider non-stationary models as in [14, Chapter 4.2].

$$
\begin{aligned}
m(\mathbf{x}) & =a_{0}+a_{1} x_{1}+a_{2} x_{2} \\
k\left(\mathbf{x}, \mathbf{x}^{\prime}\right) & =\exp \left(-\left[\frac{\left(x_{1}-x_{1}^{\prime}\right)}{\alpha_{x_{1}}}\right]^{2 / \gamma}-\left[\frac{\left(x_{2}-x_{2}^{\prime}\right)}{\alpha_{x_{2}}}\right]^{2 / \gamma}\right)
\end{aligned}
$$

The distribution parameters $a_{0}, a_{1}, a_{2}, \alpha_{x_{1}}, \alpha_{x_{2}}$, and $\gamma$ are computed from the observed data. By far the most popular technique for doing that is the one of using the maximum likelihood approach [14]. In that approach, the likelihood function is written based on the covariance function Gaussian process representation. Then, it is maximized by using standard optimization techniques. While the approach is laborious, it is also fairly straightforward, standard, and comprehensively described in multiple references, such as [14, Chapter 5]. One of the detriments of using other processes (non-Gaussian) for data-driven uncertainty quantification, is the difficulty of estimating the hyperparameters efficiently and accurately. In this work, we concentrate on the issues concerning the application of the Gaussian process model for representating the state of the road surface in conjunction with advanced dynamical simulation tools.

The phase parameter $f$ remains to be evaluated at all $n$ nodes of the evaluation grid $\mathbf{x}_{*}$.

$$
\mathbf{x}_{*}=\left(\begin{array}{c}
\left(x_{11}, x_{21}\right) \\
\vdots \\
\left(x_{1 n}, x_{2 n}\right)
\end{array}\right) \in \mathbb{R}^{n}
$$

If $\mathbf{W} \in \mathbb{R}^{m}$ is the set of observed values, a provision is made for including noise in this data by means of the parameter $\sigma_{n}$,

$$
\begin{aligned}
\overline{\mathbf{f}_{*}} & =\mathbf{m}\left(\mathbf{x}_{*}\right)+\mathbf{k}\left(\mathbf{x}_{*}, \mathbf{x}\right) \mathbf{k}_{\mathbf{W}}^{-1}(\mathbf{W}-\mathbf{m}(\mathbf{x})) \in \mathbb{R}^{n} \\
\mathbf{k}_{\mathbf{W}} & =\mathbf{k}(\mathbf{x}, \mathbf{x})+\sigma_{n}^{2} \mathbf{I} \in \mathbb{R}^{m \times m} \\
\mathbf{C O V}\left(\mathbf{f}_{*}\right) & =\mathbf{k}\left(\mathbf{x}_{*}, \mathbf{x}_{*}\right)-\mathbf{k}\left(\mathbf{x}_{*}, \mathbf{x}\right) \mathbf{k}_{\mathbf{W}}{ }^{-1} \mathbf{k}\left(\mathbf{x}, \mathbf{x}_{*}\right) \in \mathbb{R}^{n \times n},
\end{aligned}
$$

where $\mathbf{x}$ is the set of all measured point coordinates and $\mathbf{x}_{*}$ is the set of all computed point coordinates.
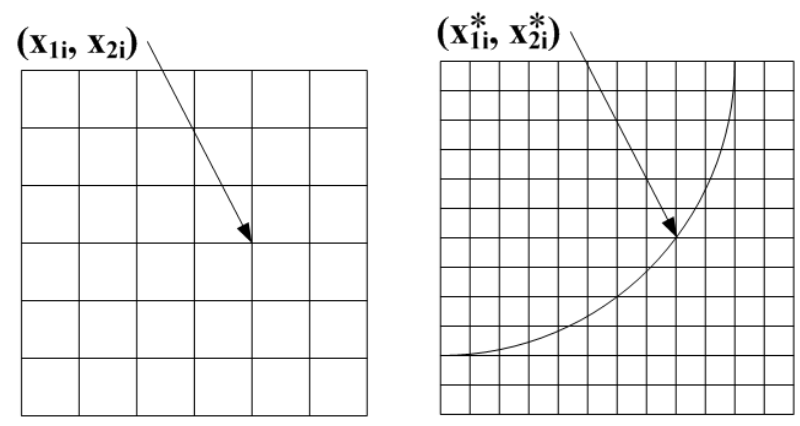

Figure 1. Gaussian processes are used to compute sampling characteristics on fine grid from deterministic data on sparse grid.

Samples at the $n$-node evaluation grid are obtained by drawing from a normal distribution with mean $\overline{\mathbf{f}}_{*}$ and covariance ma$\operatorname{trix} \operatorname{COV}\left(\overline{\mathbf{f}}_{*}\right)$ :

$$
\mathbf{f}\left(\mathbf{x}_{*}\right) \sim N\left(\overline{\mathbf{f}_{*}}, \operatorname{COV}\left(\overline{\mathbf{f}_{*}}\right)\right) \in \mathbb{R}^{n}
$$

For each sample, a cubic spline is used in conjunction with the generated data to produce friction coefficients outside the $n$-node grid. During the simulation, the spline is invoked to evaluate $f$ at all the road-tire contact points at any time, as shown in Figure 4. The equations of motion are formulated and solved by using friction coefficient input from the constructed spline. The vehicle positions and velocities are computed for each sample and averaged; furthermore, variance is computed at each simulation time step.

Of course, at points away from the evaluation grid, the field function $f$ approximated by splines no longer obeys the Gaussian process model, it is only an approximation of it. One can show analytically, however, that, in the limit of the evaluation grid spacing going to zero, the trajectories produced by the dynamical simulator converge to those that would be obtained if proper Gaussian process sampling would had been employed at the points required by the integration procedure. This convergence is due to the fact that almost any sample $f$ surface is smooth [14]. The results of a convergence study are shown in Figs. 2 and 3. First in the study, a spline was fit to an extremely fine Gaussian-distributed grid and used to run a baseline simulation. For each subsequent simulation, 3/4 of the Gaussiandistributed points were removed and a spline was fit to the remaining points and used to run a simulations. It was expected that the error of the simulation trajectories would converge to zero as the resolution went to zero. Figure 2 shows the deviation from baseline as a function of time; Figure 3 shows the convergence of the normalized error as grid resolution goes to zero. 


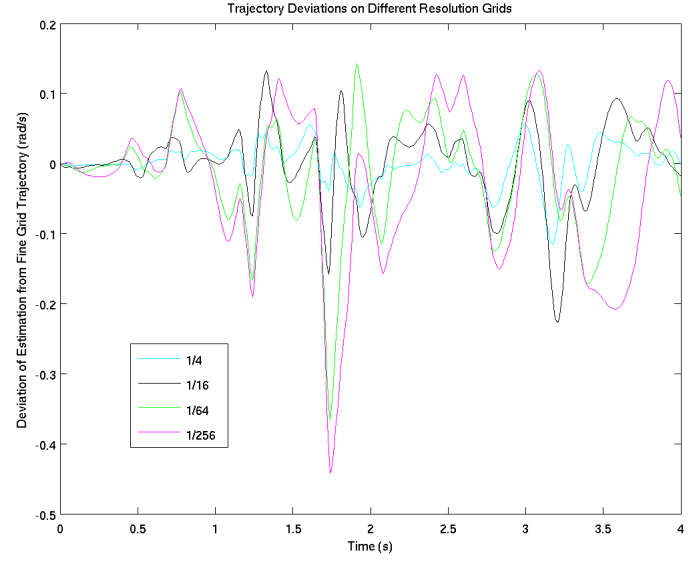

Figure 2. Deviation of trajectory from fine grid trajectory for splines fit to varying resolution grids

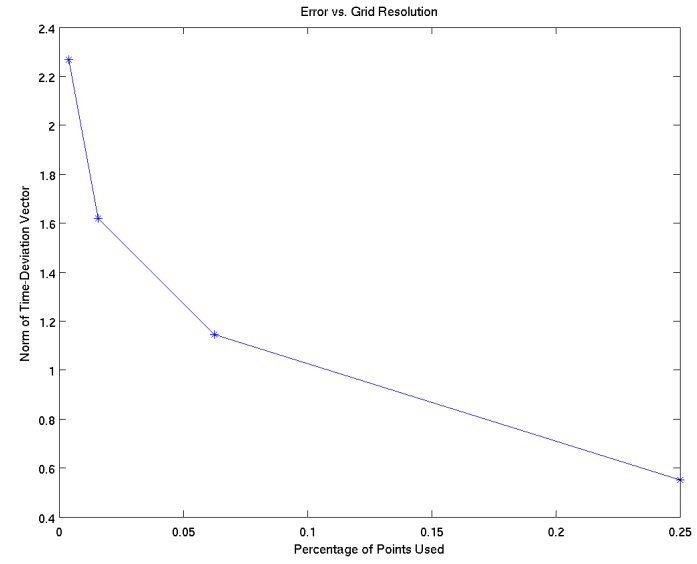

Figure 3. Convergence of trajectory as evaluation grid spacing goes to zero.

A summary of the overall methodology is presented in Figure 5. The approach starts with a specification of the variogram model adopted to capture the spatial statistical distribution of the friction coefficient. Selecting a variogram model compatible with the underlying statistics of the physical process is important, particularly so when the number of measurements is very limited. To this end, a priori knowledge and expert opinion are often relied upon in choosing the spatial variogram type. When a wealth of data is available, misspecification of the variogram, although not desirable, is acceptable, since asymptotically it gets corrected as discussed in [15-17]. When little is known about the underlying statistics of the physical process, empirical guidelines for selecting a variogram are discussed in $[18,19]$. In this case,

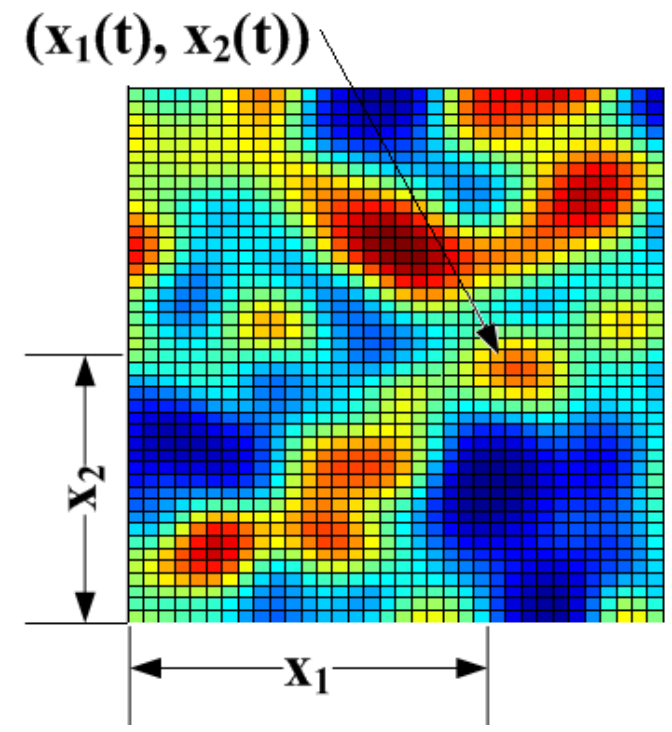

Figure 4. A spline interpolant of friction coefficient samples is extracted from for each Monte Carlo iteration.

the impact of variogram misspecification on kriging can be significant [20], and adopting a nonparametric variogram estimation technique [21-26] or relying on expert opinion are recommended options.

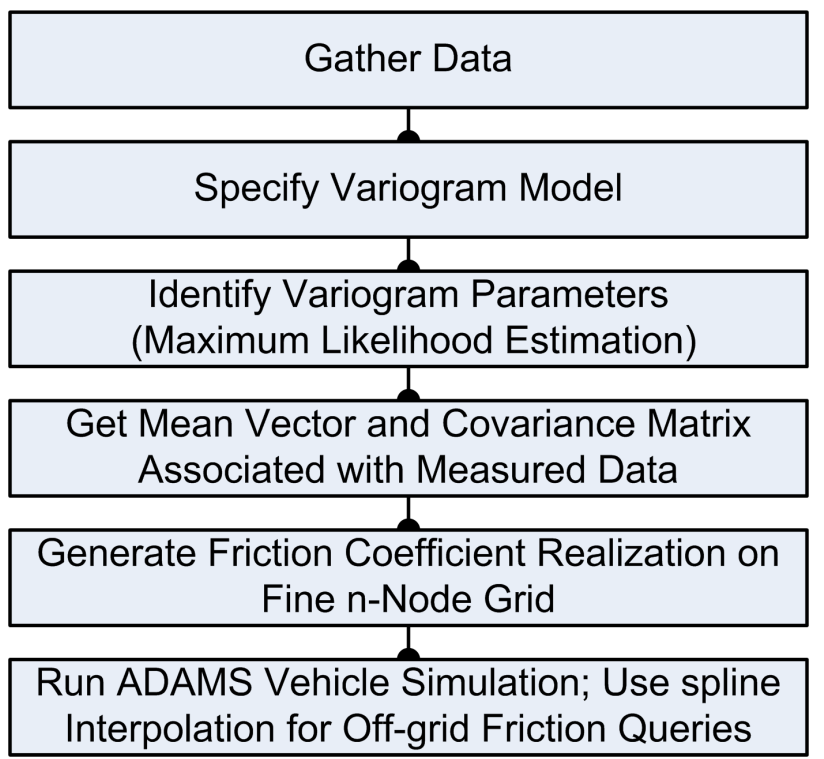

Figure 5. Overall methodology. This work concentrates on the last three stages of the methodology. Data gathering, specification of variogram, and variogram parameter identification fall outside the scope of this work. 


\section{MODELS CONSIDERED}

The first model considered is a simple bicycle model implemented in MATLAB. An open-loop step steer angle is used to negotiate a turn. A high-fidelity car model is used as well. The vehicle is modeled in MSC.ADAMS/Car and is used to perform a J-turn maneuver: drive straight up to a certain point, then apply a ramp steer input to the steering wheel. These models are presented in more detail in the following subsections.

\section{Bicycle Model}

The bicycle model, shown in Figure 6, has three degrees of freedom: longitudinal motion $V_{x}$, lateral motion $V_{y}$, and yaw $\Omega_{z}$. Three input functions determine the behavior of the model: steer angle $\delta_{f}(t)$ and the front/rear wheel road adhesion coefficients $\mu_{f}$ and $\mu_{r}$, respectively.
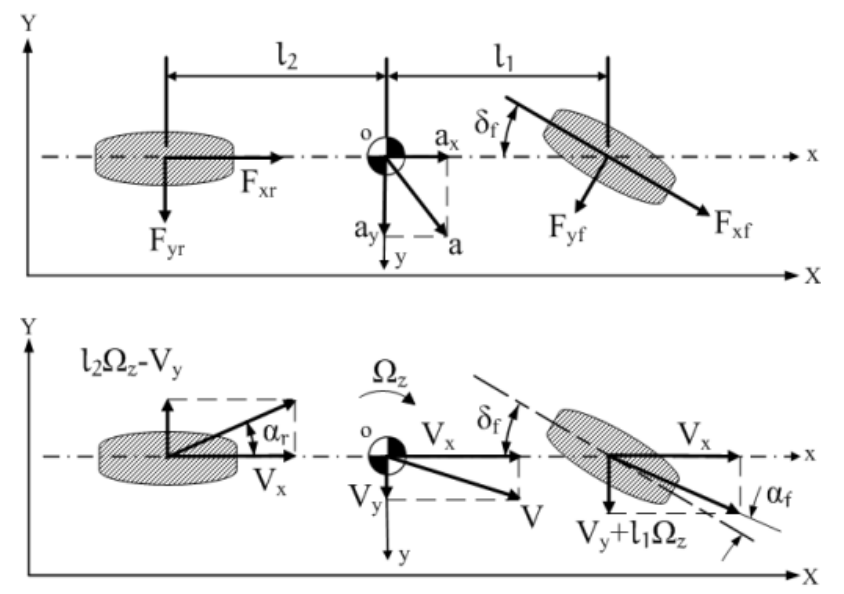

Figure 6. Bicycle model used in preliminary research of methodology [13].

After neglecting roll and asymetry and assuming that no thrust forces exist (that is, the vehicle coasts into the turn), the governing differential equations for vehicle velocities and positions are

$$
\begin{aligned}
m\left(\dot{V}_{x}-V_{y} \Omega_{z}\right) & =-F_{y f} \sin \delta_{f} \\
m\left(\dot{V}_{y}+V_{x} \Omega_{z}\right) & =F_{y r}+F_{y f} \cos \delta_{f} \\
I_{z} \dot{\Omega}_{z} & =l_{1} F_{y f} \cos \delta_{f}-l_{2} F_{y r}
\end{aligned}
$$

$$
\begin{aligned}
\dot{X} & =V_{x} \cos \Theta_{z}-V_{y} \sin \Theta_{z} \\
\dot{Y} & =V_{x} \sin \Theta_{z}+V_{y} \cos \Theta_{z} \\
\dot{\Theta}_{z} & =\Omega_{z} .
\end{aligned}
$$

The geometric parameters for the bicycle were taken from [27]. The constitutive equations for the forces acting on the tires are provided by [13].

$$
\begin{gathered}
F_{y f}= \begin{cases}\frac{\mu_{p} W_{f}}{2 \tan \alpha_{c}} \tan \alpha_{f} & \alpha_{f} \leq \alpha_{c} \\
\mu_{p} W_{f}\left(1-\frac{\tan \alpha_{c}}{2 \tan \alpha_{f}}\right) & \alpha_{f}>\alpha_{c}\end{cases} \\
F_{y r}= \begin{cases}\frac{\mu_{p} W_{r}}{2 \tan \alpha_{c}} \tan \alpha_{r} & \alpha_{r} \leq \alpha_{c} \\
\mu_{p} W_{r}\left(1-\frac{\tan \alpha_{c}}{2 \tan \alpha_{r}}\right) & \alpha_{r}>\alpha_{c}\end{cases}
\end{gathered}
$$

$W_{f}$ and $W_{r}$ are the front and back tire normal forces, respectively; $\alpha$ is the respective slip angle for each tire; $\mu_{p}$ is the respective peak road adhesion coefficient for each tire; and $\alpha_{c}$ is the critical slip angle.

Geometrically, the slip angles are related to the state variables and the steer angle alone.

$$
\begin{gathered}
\alpha_{f}=\delta_{f}-\arctan \frac{l_{1} \Omega_{z}+V_{y}}{V_{x}} \\
\alpha_{r}=\arctan \frac{l_{2} \Omega_{z}-V_{y}}{V_{x}}
\end{gathered}
$$

\section{ADAMS Car Model}

The more sophisticated vehicle model is obtained through MSC.ADAMS/Car, a full vehicle simulation package distributed by MSC.Software. The vehicle parameters used were taken directly from the default MSC.ADAMS/Car library. The full vehicle model is the integration of several subsystems including a rack-and-pinion type steering subsystem, an Ackerman arm suspension system, and a flexible chassis. Figure 7 shows the topology of a vehicle with front and rear suspension, wheels, and steering subsystems (the chassis is not shown).

The test rig is a special subsystem that conveys user inputs for steering angle to the model. ADAMS/View variables called "Communicators" are used to communicate between the subsystems. 


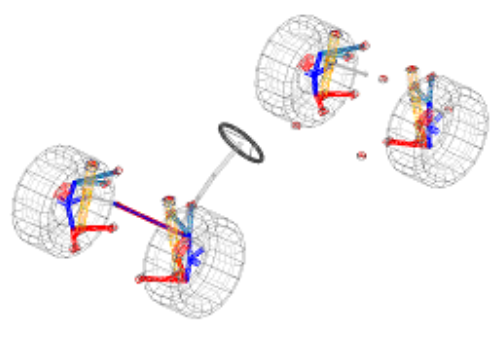

Figure 7. Vehicle model without chassis shown in ADAMS.

Because load transfer through the tires to the ground is essential in predicting vehicle travel on ice, a sophisticated and robust tire model, FTire (Flexible Ring Tire [28]) is employed in the simulation. The tire ring is numerically approximated by a finite number of discrete masses called belt elements coupled with their direct neighbors by stiff springs with in- and out-of-plane bending stiffnesses. The method is summarized by the schematic in Figure 8.
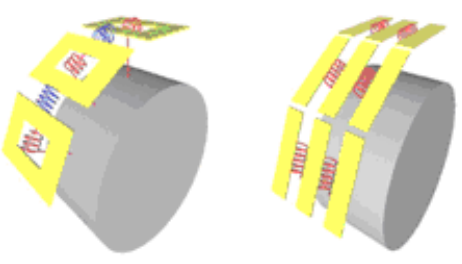

Figure 8. FTire modeling approach.

The driver used in the ADAMS/Car simulation is also openloop. The car starts at the grid origin with an initial velocity and is allowed to coast in a straight line for 0.5 seconds. Then a forty degree per second ramp function is imposed on the steering wheel for the duration of the simulation to simulate a left-hand turn.

In order to represent the road in ADAMS/Car, a custom 3D road file was created. The road file is a flat, rectangular surface tessellated with identical right triangles. The legs of the right triangles have a length of one meter matching the resolution of the computed grid $\mathbf{x}_{*}$; thus, the coordinates of the Gaussian processes' computed grid match the nodal coordinates of the ADAMS/Car road file. The friction coefficient of each triangle is the geometric average of the friction coefficients at the respective triangle vertices. A unique road file is created for each Monte Carlo iteration. The FTire model previously discussed computes the interfacial friction coefficients at each step in simulation, and the input is used to determine the vehicle dynamics.

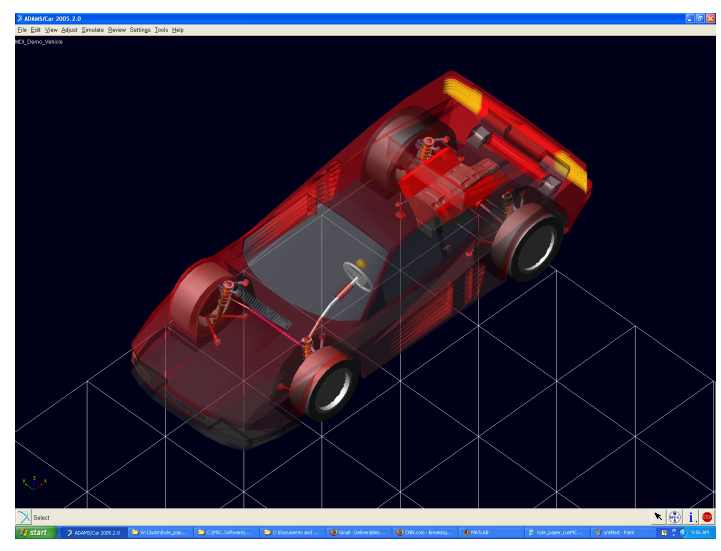

Figure 9. Car model and tessellated road surface in the ADAMS environment.

\section{NUMERICAL EXPERIMENTS}

Numerical experiments have been conducted and are presented to show that average vehicle dynamics are predicted through our methodology, verify that dynamics trends produced through full vehicle simulation in different conditions are congruous with expectations, and illustrate the insights provided by the stochastic analysis enabled by the proposed methodology. MSC.ADAMS/Car results are presented to illustrate the readiness of this methodology for industry applications. Simulation performance metrics also are given to clarify the potential of this methodology for real-time simulation.

\section{Ice Models}

The Gaussian process-based approach outlined is used to create a set of grids $G$, where each grid has a distribution reflective of the observed ice distribution. That is, the grids in $G$ are distinct but consistent in that they share the same spatial distribution characteristics (with some noise associated with the friction measurement devices). Each grid in $G$ is subsequently used for a Monte Carlo analysis, and it should have between 100 and 1000 nodes to attain accuracy yet maintain efficiency. Three grids from $G$ are shown in Figure 10; note that the grids are unique but possess comparable amplitudes and spatial variations.

The key distribution characteristics extracted from the data are the spatial variances, or literally mean patch lengths $\left(\alpha_{x_{1}}\right.$ and $\alpha_{x_{2}}$ in Eq. 4.). The reason is that rapid changes in friction coefficients result in a lateral force unbalance between the front and back tires on a vehicle resulting in understeer or oversteer [27]. 


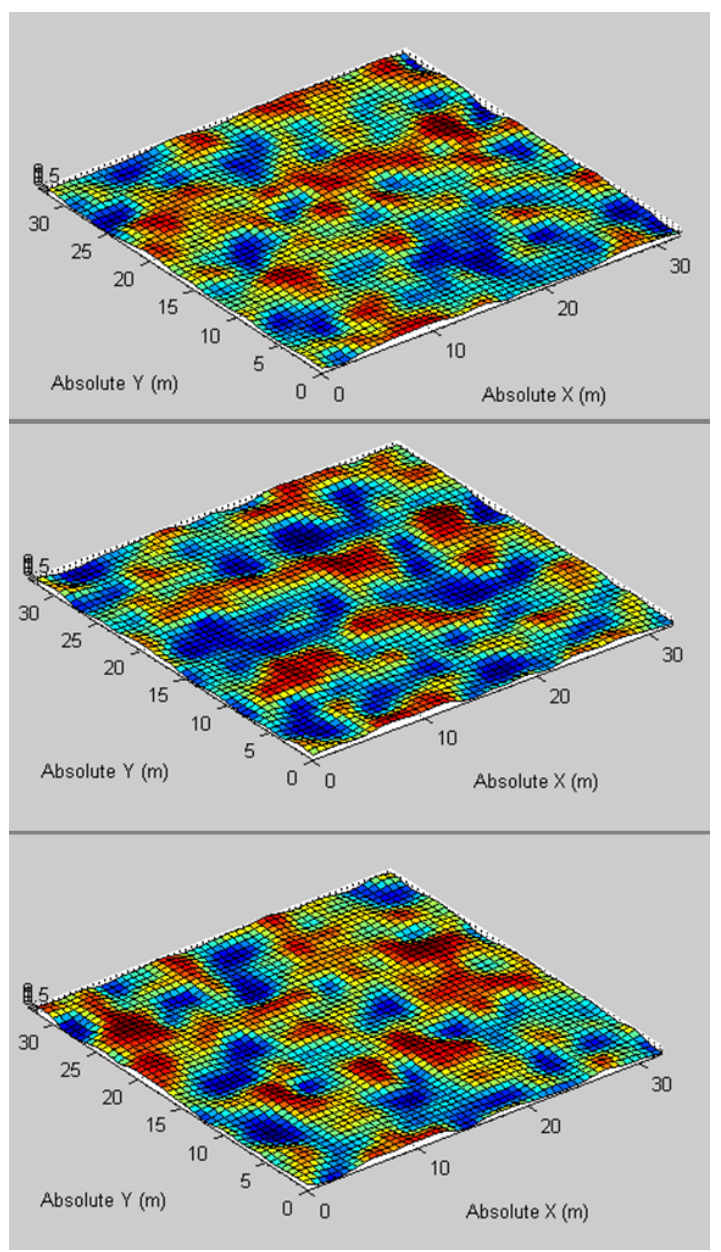

Figure 10. Phase parameter grids created from the same set of observed data.

The grids shown in Figure 11 are from two different sets of observed data: the top plot from a mean patch length of one meter and the bottom plot from a mean patch length of three meters. These grids demonstrate the sensitivity of the methodology to spatial variance.

As indicated earlier, in order to account for the biextremal nature of the friction coefficient, a phase parameter $f-$ the $\log$ arithm of a ratio involving the friction coefficient - is used. The Gaussian process is exercised on the phase parameter distribution, creating a phase parameter grid that is subsequently transformed to a friction coefficient grid. Figure 12 shows a realization of $f$ on a grid and the corresponding realization of $\mu$ on the same grid.

The grids used in the following simulations were produced from randomly generated data assuming the values of distribution parameter from Eqs. 3 and 4: $a_{0}=a_{1}=a_{2}=0, \gamma=1$, and $\sigma_{n}=.15$. Different parameters, $\alpha_{x_{1}}$ and $\alpha_{x_{2}}$, were used in experimentation

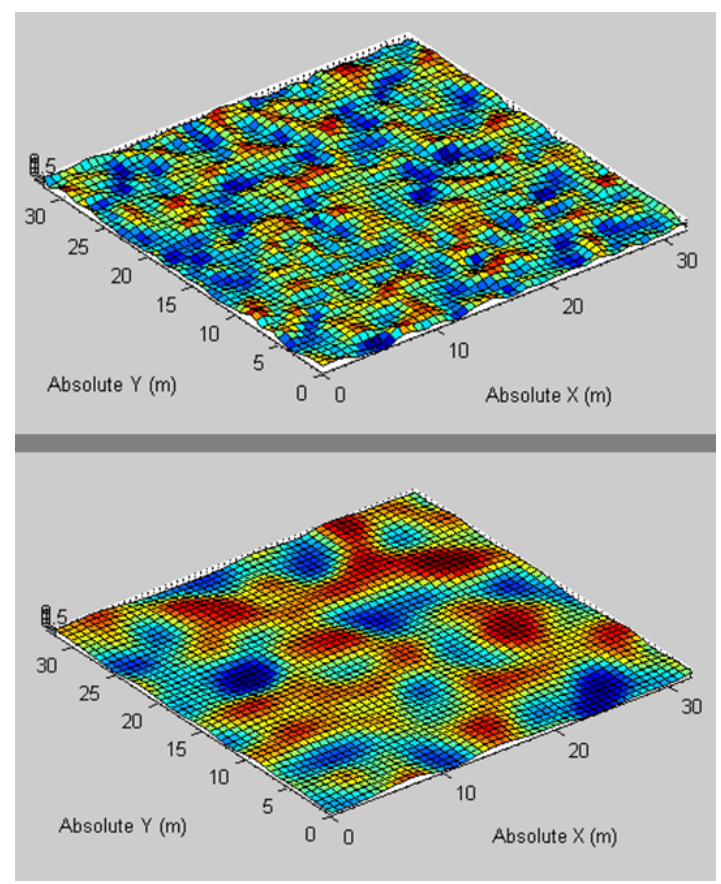

Figure 11. Phase parameter grids created from data sets with mean patch lengths one meter (top) and three meters (bottom).

and are stated for individual tests as mean patch lengths.

\section{Bicycle Simulation}

The bicycle dynamics were investigated in MATLAB, and two simulation outcomes were monitored: yaw velocity, to gauge spin-out and instability, and global position, to gauge deviation from the desired path as a product of slip, oversteer, or understeer. Simulations were first run with deterministic conditions, and Figures 13 and 14 show the friction input to each bicycle tires and the yaw velocity output as functions of time, respectively. The greatest instabilities occur during rapid friction changes; the high yaw rates are reached when the front steering tire has more traction than the rear tire (particularly between 13 and 14 seconds in simulation time).

Gaussian processes were implemented and simulations run for several vehicle conditions including a variety of speed and steer angles. Different data sets were used to represent road conditions. The simulation results shown in Figs. 15 and 16 are for high and low ice densities, respectively. Several interesting similarities exist between the two simulations and across the other simulations conducted. First, the average response (dynamics) of the vehicle is far from the constant friction case; this disparity results in deviation from the desired travel path and makes navigation more difficult. Second, as shown in Figure 17, the uncertainty in the response tends to increase in time or with distance 


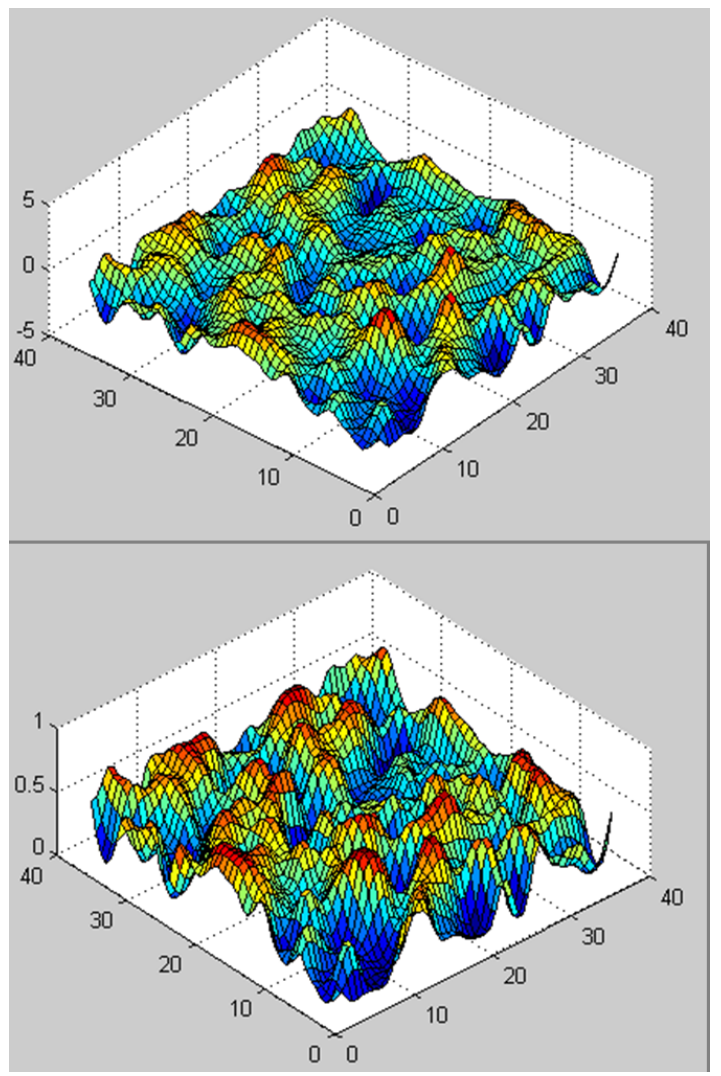

Figure 12. Transformation from the phase parameter grid (top) to the friction coefficient grid (bottom).

traveled. This illustrates the danger of long turns if the driver is not vigilant at the wheel. As the driver progresses around the turn, the risk of instability increases. Third, the uncertainty of the vehicle dynamics is varying in space, as seen in Figure 17. We have noticed that deep local minima in the yaw uncertainty correspond to passing near a known data point as verified by comparing the global position at these time iterations with the observed data coordinates. That is perhaps to be expected, though the depth of some of those minima was surprising to us given that for dynamical systems the uncertainty tends to grow fairly steadily in time. Whether higher certainty of a surface patch state can be exploited in a control procedure is an interesting topic for future research.

An experiment was set up to validate the predictive capability of the methodology. The original random set of friction coefficients was amended to introduce a strip of abrupt ice (low friction coefficients) approximately two seconds into the vehicle's travel. The result of the simulation is shown in Figure 18; the constant friction simulation would have resulted in a yaw velocity of approximately 0.2 but it is not shown for the sake of clarity. Clearly, the Gaussian process accounts for the ice strip

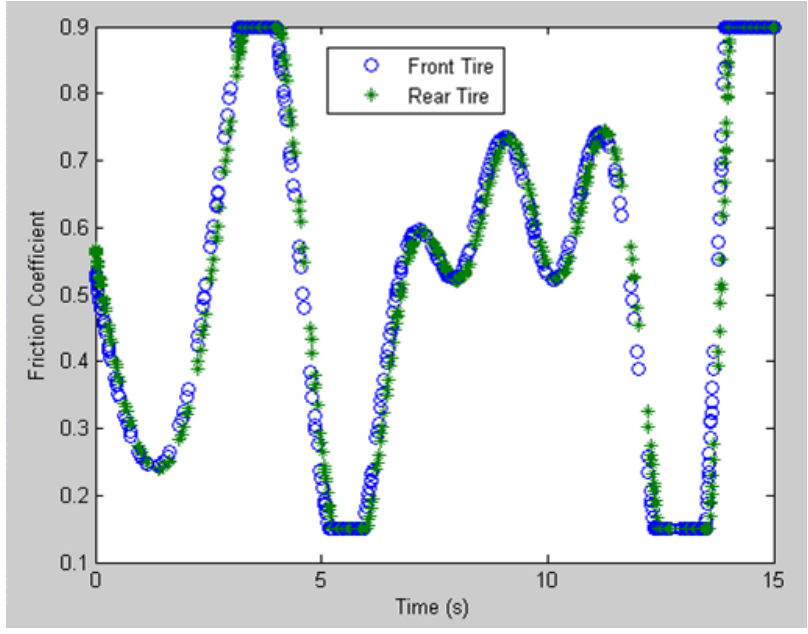

Figure 13. Deterministic simulation: friction coefficient input.

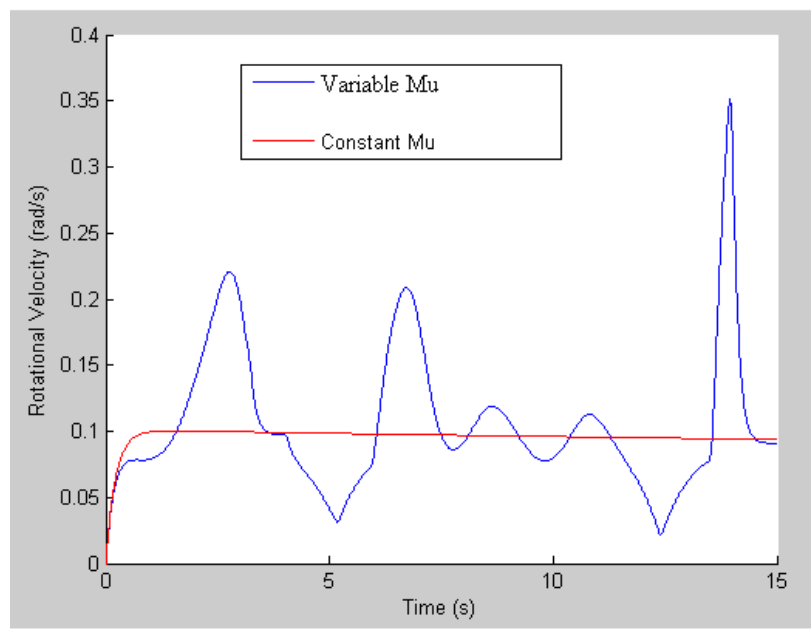

Figure 14. Deterministic simulation: resulting vehicle dynamics.

as nearly all Monte Carlo iterations diverge drastically from the constant friction dynamics. The resulting average indicates that the driver will experience an uncomfortable change in yaw velocity and should enter the turn at a lower speed. Incidentally, the drop in yaw around 3.5 seconds was the result not of a manual ice insertion but rather of a coincidental low friction coefficient grouping generated randomly.

\section{ADAMS Car Simulation}

To demonstrate the propensity of our methodology for industry applications, we introduced our ice model into MSC.ADAMS/Car. The car model used considers several vehicle subsystems and intersystem interactions to produce high- 


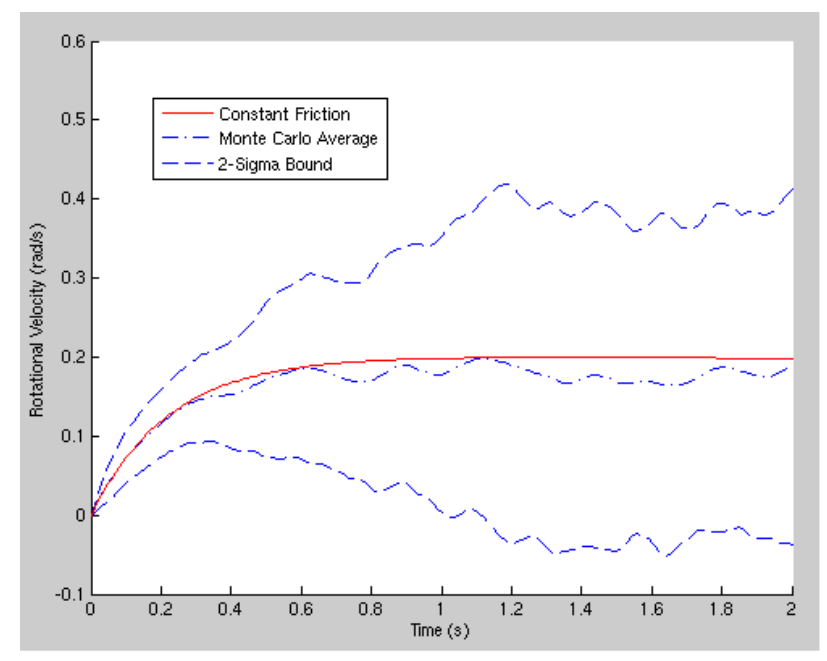

Figure 15. Bicycle simulation: one-meter mean patch length, one degree steer angle, 200 Monte Carlo iterations.

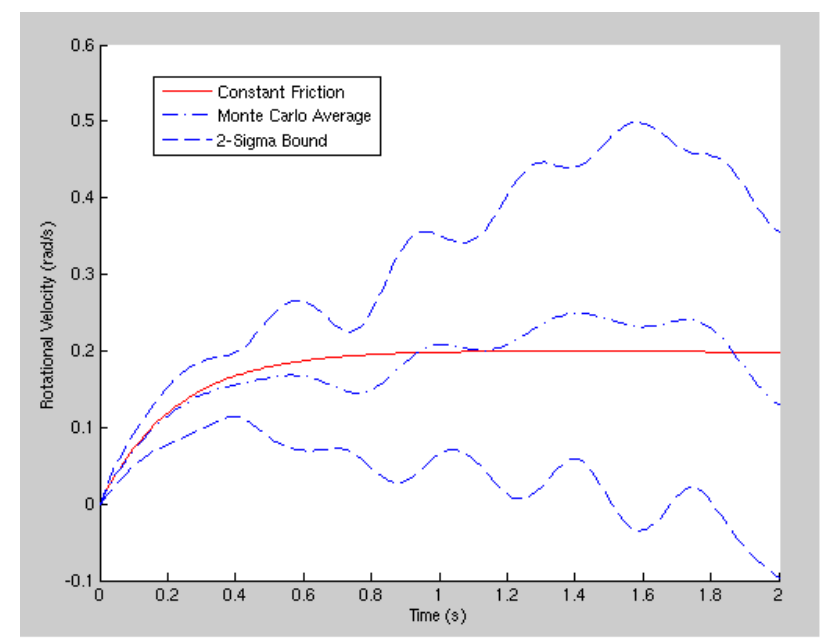

Figure 16. Bicycle simulation: three-meter mean patch length, one degree steer angle, 200 Monte Carlo iterations.

fidelity results as explained in the Models Considered section. The results shown in Figs. 19 and 20 are for the simulation of a car executing a left turn on an icy road. The observed friction data, for the results shown, was generated randomly and then manipulated to introduce a strip of ice expectedly two seconds into the vehicle's travel. The Gaussian process results in Figure 19 possess the same three characteristics discussed in the Bicycle model results: divergence from the constant friction case, proliferation of uncertainty with time, and alternating uncertainty depending on proximity to observed data coordinates. The paths of travel shown in Figure 20 are one example of how our method-

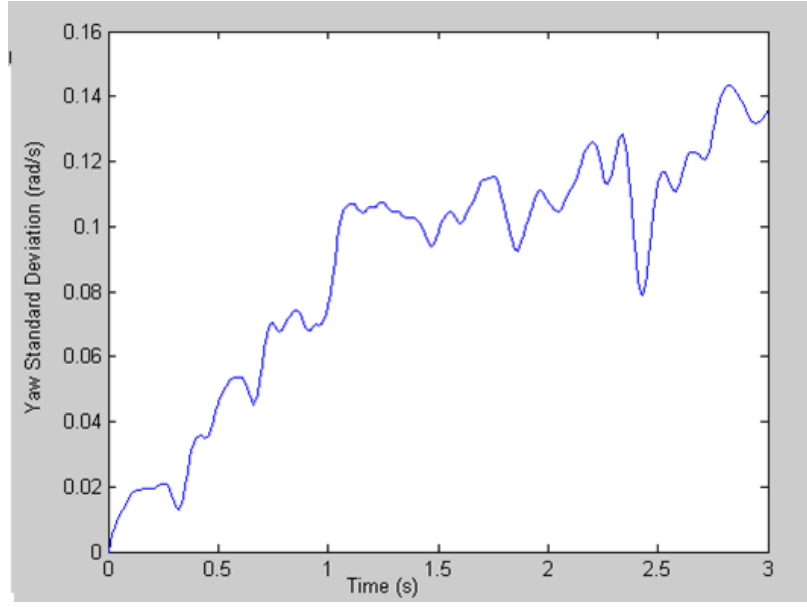

Figure 17. Dynamical uncertainty as a function of time: one meter patch length, one degree steer angle, 200 Monte Carlo iterations.

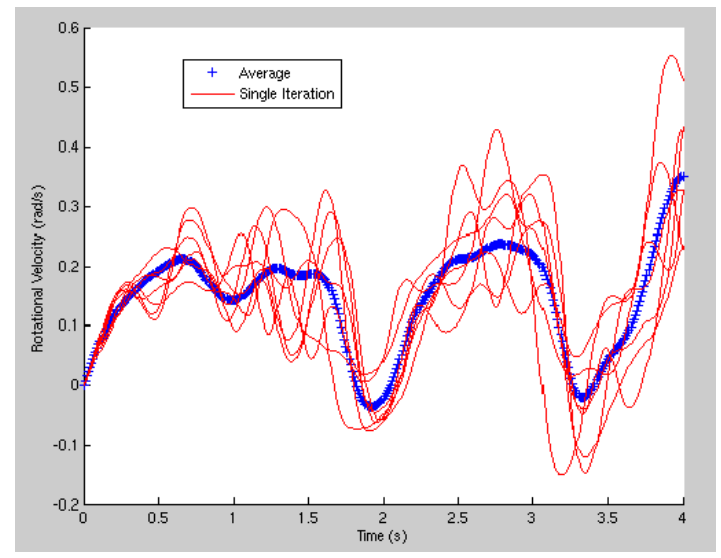

Figure 18. Gaussian process simulation with implanted ice strip: threemeter mean patch length, one degree steer angle, 200 Monte Carlo iterations.

ology could be an asset to visualization and communication in industry. Moreover, the divergence from the desired turn is the result of the strip of ice in the road and possibly other smaller patches accumulated in the randomly generated data; quantifying the magnitude of this divergence is essential to driver safety.

\section{Diagnostics}

For this methodology to be useful in an industry setting, it has to produce results fast and reliably. To understand the runtime characteristics of the simulation processes, we monitor the duration of generating a realization $\mathbf{x}_{*}$ for different simulation times (Figure 21), different evaluation grid $\left(\mathbf{x}_{*}\right)$ resolutions (Figure 22), and different sample grid ((x) resolutions (Figure 23). 


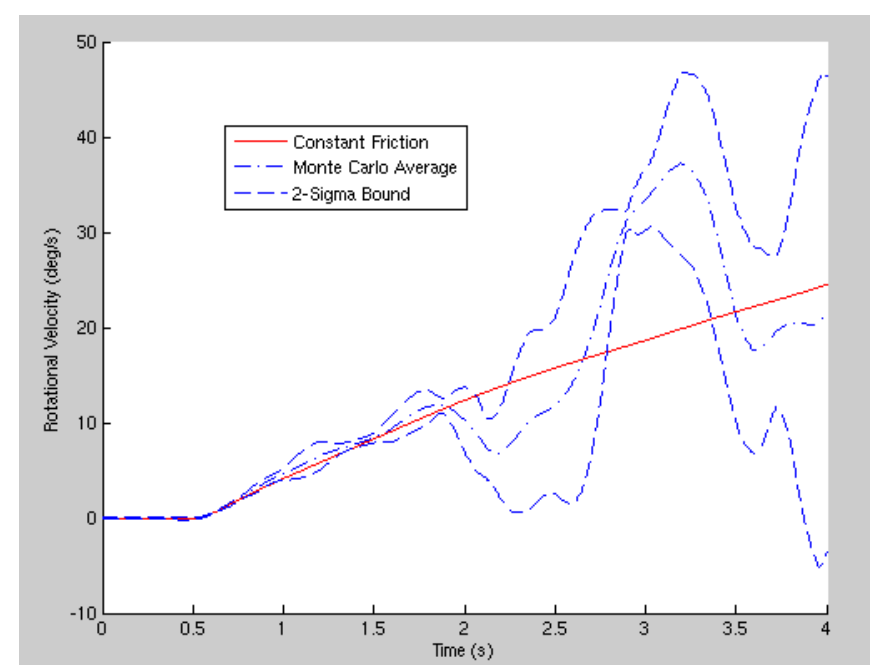

Figure 19. Yaw velocity vs. time for ADAMS ramp steer simulation: three-meter mean patch length, forty degree/second ramp steer, 5 Monte Carlo iterations.

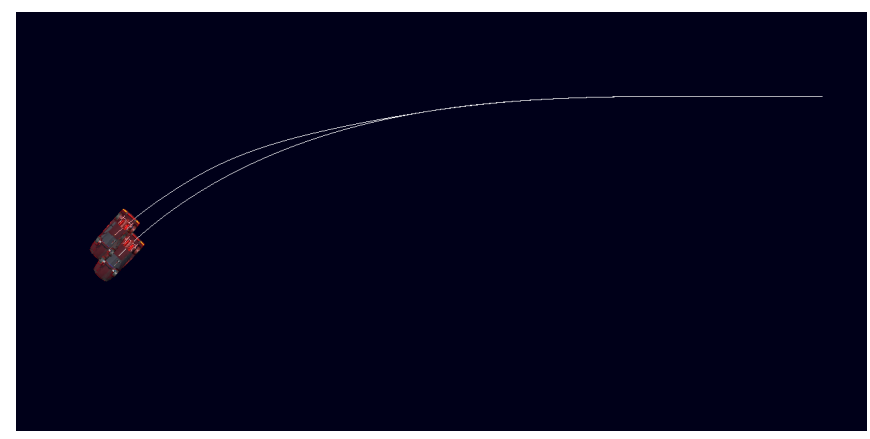

Figure 20. Path of travel for ADAMS ramp steer simulation: three-meter mean patch length, fourty degree/second ramp steer, 5 Monte Carlo iterations.

The results should be considered qualitatively because of the diversity in computing systems across industry. We see that the most influential variable to total runtime is length of the maneuver. As the maneuver gets longer, it requires that the surveyed and computed space increase, increasing the dimensions of the matrices involved in computation (see Eqs. 6,7 and 8). Hence, runtime increases dramatically. Real-time simulation can be achieved for short periods (5 to 10 second maneuvers), but the current methodology proves to be inadequate for longer simulations.

The plots for runtime vs. grid size demonstrate that the runtime bottleneck occurs with finer $\left(\mathbf{x}_{*}\right)$ resolutions but not finer $(\mathbf{x})$ resolutions. Hence, one must be careful when selecting evaluation grid sizes; a balance should be found between runtime and

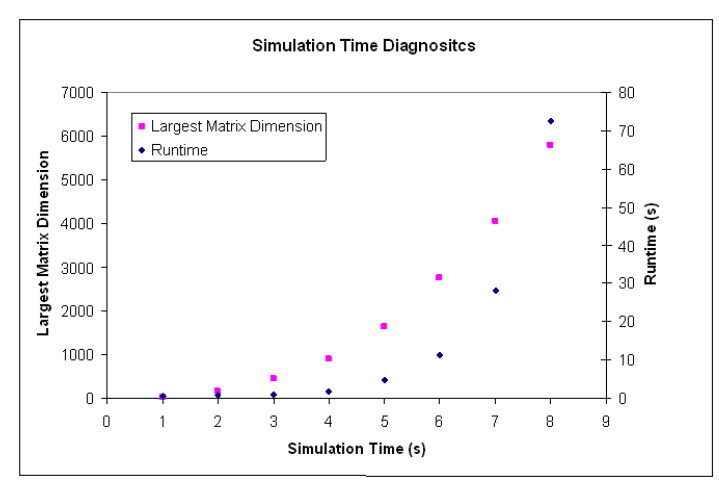

Figure 21. Runtime and largest matrix size as a function of simulation time.

accuracy. This is a topic of ongoing research.

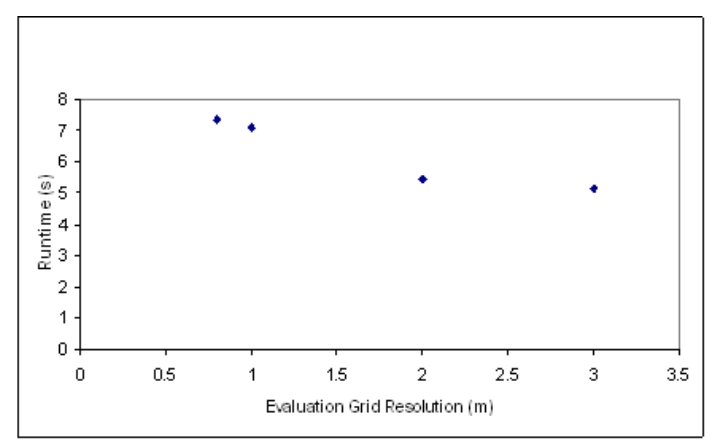

Figure 22. Runtime as a function of evaluation grid $\left(\mathbf{x}_{*}\right)$ size.

\section{CONCLUSIONS AND FUTURE WORK}

This paper outlines a framework for uncertainty quantification in vehicle dynamics simulation. The methodology draws on a Gaussian process model and maximum likelihood estimation to capture in a consistent way uncertainty that enters the dynamics of a complex vehicle model represented in ADAMS. Unlike other approaches such as Galerkin-based polynomial chaos, the proposed framework enables a black box setup in which the software package used to solve for the dynamics (time evolution) of the mechanical system requires no modification. In addition, the representation of the model is far less complex compared to the polynomials chaos expansions, of either Galerkin and colocation types. We use a covariance function-based Gaussian process modeling approach. It has the advantage of being more flexible than previous, spectral-based Gaussian process approaches, as well as being one of the prevalent approaches in geostatistics, 


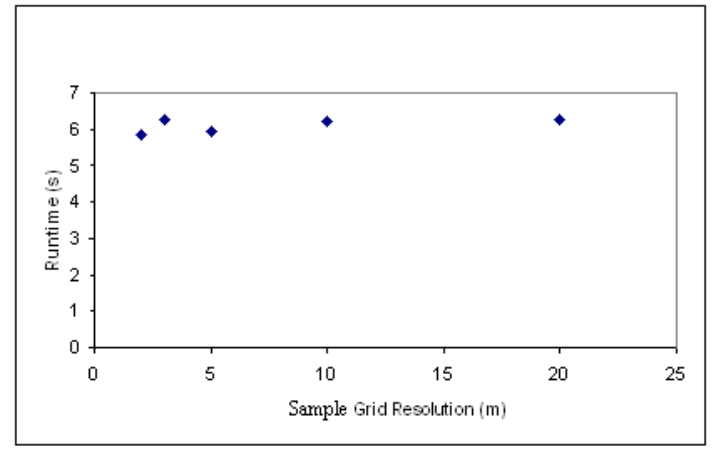

Figure 23. Runtime as a function of sample grid $(\mathbf{x})$ size.

making it likely that this approach will be easy to use in conjunction with emerging spatial database technology. Insofar as simulation, once the Gaussian process model over the road surface is obtained, the methodology relies on a Monte Carlo step that generates the information required to produce the vehicle dynamics statistics of interest.

Several steps have not been discussed here but are being addressed in ongoing projects. First, work is underway to extend the Gaussian process-based uncertainty model to other classes of models, including nonstationary models. Since in kriging the choice of weights is completely determined by the choice of the variogram model, it is particularly important for handling spatial uncertainty (road-tire friction coefficients, road elevation) to look at other models beyond Gaussian processes. Since a Gaussian process approach is guaranteed to work provided the amount of measured data is large, other questions of interest are how to handle effectively large sets of measurements and how to use consistently and update periodically subsets of data to handle only subregions of interest. The latter would allow one to handle smaller regions of data that are surrounding the vehicle as it moves on a road.

The methodology presented was illustrated for an application where the source of uncertainty is provided by the road/tire friction coefficient. It remains to investigate how road profile uncertainty reflects in the overall vehicle dynamics. Such an undertaking critically depends on the quality of the tire models used in the vehicle simulation. However, the FTire model relied on in this work provides a level of fidelity for fully three-dimensional simulation that makes terrain-uncertainty investigation possible and likely to be very insightful.

\section{ACKNOWLEGMENTS}

Dr. Bahram Fatemi and Dr. Reza Shadeghi are gratefully acknowledged for facilitating the research support coming from British Aerospace Engineering (BAE) and MSC.Software, respectively, that made possible the portion of this work carried out in the Simulation Based Engineering Lab at the University of Wisconsin, Madison. Mihai Anitescu was supported by Contract No. DE-AC02-06CH11357 of the U.S. Department of Energy.

\section{REFERENCES}

[1] Robson, J., and Dobbs, C., 1975. "Stochastic road inputs and vehicle response". Vehicle System Dynamics, 5, pp. 13.

[2] Narayanan, S., 1990. "Stochastic optimal-control of nonstationary response of a single degree-of freedom vehicle model". Journal of Sound and Vibration, 141, pp. 449-463.

[3] Hammond, J. K., and Harrison, R. F., 1984. Covariance equivalent forms and evolutionary spectra for nonstationary random processes, Vol. 62. Springer Berlin/Heidelberg.

[4] Rustighi, E., and Elliot, J. S., 2007. "Stochastic road excitation and control feasibility in a $2 \mathrm{~d}$ linear tyre model". Journal of Sound and Vibration, 300, pp. 490-501.

[5] Lei, X., and Noda, N., 2002. "Analyses of dynamic response of vehicle and track coupling system with random irregularity of track vertical profile". Journal of Sound and Vibration, 258, pp. 147-165.

[6] Karacay, T., Akturk, N., and Eroglu, M., 2004. "Nonstationary response of a vehicle obtained from a series of stationary responses". KSME International Journal, 18, pp. $1565-1571$.

[7] Wallman, C., and Astrom, H., 2001. "Friction measurement methods and the correlation between road friction and traffic safety. a literature review.". VTI meddelande 911A.

[8] Holzmann, F., Bellino, M., Siegwart, R., and Bubb, H., 2006. "Predictive estimation of the road-tire friction coefficient". In Control Applications IEEE International Conference.

[9] Rajamni, R., Piyabongkarn, D., Lew, J., and Grogg, J., 2006. "Algorithms for real-time estimation of individual wheel tire-road friction coefficients". In American Control Conference.

[10] MSC.Software, 2005. ADAMS User Manual. Available online at http://www.mscsoftware.com.

[11] Chiles, J. P., and Delfiner, P., 1999. Geostatistics: modeling spatial uncertainty. Wiley, New York.

[12] Stein, M., 1999. Interpolation of Spatial Data: Some Theory for Kriging. Springer.

[13] Wong, J., 2001. Theory of Ground Vehicles. John Wiley \& Sons, Inc.

[14] Rasmussen, C., and Williams, C., 2006. Gaussian Processes for Machine Learning. MIT Press.

[15] Stein, M., 1988. "Asymptotically efficient prediction of a random field with a misspecified covariance function". Ann. Stat, 16, pp. 55-63.

[16] Stein, M., 1990. "Uniform asymptotic optimality of lin- 
ear predictions of a random field using an incorrect secondorder structure". Ann. Statist, 18(2), pp. 850-872.

[17] Stein, M., and Handcock, M., 1989. "Some asymptotic properties of kriging when the covariance function is misspecified". Mathematical Geology, 21(2), pp. 171-190.

[18] Journel, A., and Huijbregts, C., 1978. "Mining Geostatistic". Academic, San Diego.

[19] Clark, I., 1979. Practical geostatistics. Applied Science Publishers, London.

[20] Gorsich, D., and Genton, M., 2000. "Variogram Model Selection via Nonparametric Derivative Estimation". Mathematical Geology, 32(3), pp. 249-270.

[21] Shapiro, A., and Botha, J., 1991. "Variogram fitting with a general class of conditionally nonnegative definite functions.". Computational Statistics \& Data Analysis, 11(1), pp. 87-96.

[22] Lele, S., 1995. "Inner product matrices, kriging, and nonparametric estimation of variogram". Mathematical Geology, 27(5), pp. 673-692.

[23] Cherry, S., 1996. "An evaluation of a non-parametric method of estimating semi-variograms of isotropic spatial processes". Journal of Applied Statistics, 23(4), pp. 435449.

[24] CHERRY, S., 1997. "NON-PARAMETRIC ESTIMATION OF THE SILL IN GEOSTATISTICS". Environmetrics, 8(1), pp. 13-27.

[25] Ecker, M., and Gelfand, A., 1997. "Bayesian variogram modeling for an isotropic spatial process". Journal of Agricultural, Biological and Environmental Statistics, 2(4), pp. 347-369.

[26] Genton, M., and Gorsich, D., 2002. "Nonparametric variogram and covariogram estimation with Fourier-Bessel matrices". Computational Statistics and Data Analysis, 41(1), pp. 47-57.

[27] Cossalter, V., 2002. Motorcycle Dynamics. Race Dynamics Inc.

[28] Gipser, M., 2005. "Ftire: a physically based applicationoriented tyre model for use with detailed mbs and finiteelement suspension models". Vehcile System Dynamics, $\mathbf{4 3}$ (supplment), pp. 76-91.

The submitted manuscript has been created in part by
UChicago Argonne, LLC, operator of Argonne National Lab-
oratory ("Argonne"). Argonne, a U.S. Department of Energy
Office of Science laboratory, is operated under Contract No.
DE-AC02-06CH11357. The U.S. Government retains for it-
self, and others acting on its behalf, a paid-up nonexclusive,
irrevocable worldwide license in said article to reproduce, pre-
pare derivative works, distribute copies to the public, and per-
form publicly and display publicly, by or on behalf of the Gov-
ernment. 